\title{
Multiple drivers of seasonal change in PRI: Implications for photosynthesis
}

\section{Leaf level}

Anatoly A. Gitelson ${ }^{\varepsilon^{*}}$, John A. Gamon ${ }^{\mu \beta}$, Alexei Solovchenko ${ }^{\phi}$

\begin{abstract}
The goal of this study was to explore the relationships between the photochemical reflectance index (PRI) at the leaf level and pigment pools, focusing on the constitutive role of pigments in influencing PRI over seasonal or ontogenetic time frames. The purpose was to reevaluate the role of PRI as an indicator of seasonally shifting pigment (chlorophyll, carotenoid and anthocyanin) contents, and hence photosynthetic activity, across a range of tree and crop species. We studied natural vegetation - three tree species (maple, chestnut and beech) and two managed irrigated and rainfed crop species (maize and soybean), contrasting in photosynthetic
\end{abstract}


24 pathway and leaf structure, and having wide variation of pigment content and composition. In 25 anthocyanin-free leaves, PRI related to both total chlorophyll (Chl) and carotenoid (Car)

26 contents, however, much closer relationships were found between PRI and Car to Chl ratio

27 (Car/Chl). The sensitivity of PRI to Car/Chl varied widely in tree species with the degree of secondary carotenoids. In crop leaves where the Car vs. Chl relationship was very close, the

29 slopes of PRI vs. Car/Chl relationships for maize and soybean were almost identical. PRI vs.

30 Car/Chl relationships for leaves of different tree species formed a significant, uniform

31 relationship with PRI. Two crops also formed a significant, uniform PRI vs. Car/Chl relationship 32 with a slope half the value found for trees. In anthocyanin-containing leaves, PRI did not clearly

33 relate to any pigment content because reflectance values at both PRI wavebands are affected by

34 anthocyanin content. The findings of a strong link between leaf level PRI and Car/Chl over

35 seasonal and ontogenetic time spans supports recent findings calling for a more careful

36 evaluation of the relationship between PRI and either LUE or photosynthetic activity. In

37 particular, studies that contrast short-term (e.g. diurnal) vs. long-term (e.g. seasonal) pigment,

38 PRI, and photosynthetic responses in contrasting vegetation types are needed.

Key words: chlorophyll, carotenoids, anthocyanin, xanthophyll cycle.

\section{Introduction}

The photochemical reflectance index (PRI) was originally derived as an optical indicator of 44 xanthophyll cycle activity and photosynthetic light-use efficiency (LUE) over diurnal time scales

45 (Gamon et al., 1992, 1993, Peñuelas et al., 1995). More recently, a large body of research has

46 demonstrated that PRI is also strongly influenced by pigment pool sizes, particularly in studies 
47 spanning seasons (Stylinski et al., 2002, Filella et al., 2009), multiple species (Sims and Gamon,

48 2002, Garrity et al., 2011, Gamon and Berry, 2012) or canopy positions (Gamon et al., 2001,

49 Gamon and Berry, 2012). These two facets of PRI that reflect the rapid xanthophyll cycle

50 activity and the slower, seasonal or ontogenetic pigment pool size adjustments have been termed

51 the "facultative" and "constitutive" responses, respectively (Gamon and Berry, 2012, Wong and

52 Gamon, 2015A, 2015B). Both are of interest because they represent physiological adjustments of

53 photosynthesis to prevailing environmental conditions, one over short (minutes or diurnal) time

54 spans, and another over long (ontogenetic or seasonal) time spans. Both have been shown to

55 correlate with light-use efficiency, but the mechanistic explanations of these correlations are

56 likely to be different (Sims and Gamon, 2002, Solovchenko, 2010, Gamon, 2015). This dual

57 nature of PRI along with different adjustments of the photosynthetic pigments occurring over

58 disparate time scales has led to considerable confusion in the PRI literature. Most remote sensing

59 studies of PRI have not directly tested mechanism at the level of pigment responses, assuming

60 implicitly that the xanthophyll cycle operation is the primary driver of the changing PRI signal.

The issue of the underlying mechanism(s) driving PRI responses has a larger significance.

62 While the majority of studies report positive PRI-LUE correlations for individual circumstances

63 (Garbulsky et al., 2011), some studies suggest that the PRI-LUE relationship is not constant

64 across all locations (Nichol et al., 2002, Goerner et al., 2011) and ontogenetic stages

65 (Solovchenko et al., 2010), and it is likely that the different underlying mechanisms discussed

66 above could be partly responsible. Understanding the mechanistic relationships between PRI,

67 LUE, and photosynthetic activity across a range of time scales is clearly important for

68 developing accurate, predictive photosynthesis models from remote sensing approaches

69 involving pigment-based reflectance indices. 
Much of the recent carotenoid (Car) research in higher plants has focused on the role of the

71 Car involved in operation of xanthophyll cycle pigments (specifically, violaxanthin,

72 antheraxanthin and zeaxanthin) in the regulation of light energy conversion and photoprotection

73 under stress. This "downregulation" of photosynthesis is balanced against the need for light

74 harvesting and photochemical utilization, served predominantly by lutein and, to a certain extent,

$75 \beta$-carotene. Another photoprotective function is served by Car and other pigments that are not

76 directly involved in the xanthophyll cycle or core photosynthetic light reactions but operate

77 mainly via light screening (for reviews, see Solovchenko, 2010, 2013). All of these pigments can

78 influence the vegetation reflectance spectra (and hence PRI). It is conceivable that the PRI

79 response would reflect the diversity of functional roles of Car in plants, particularly in the

80 context of different plant types having different pigment responses.

Recently, Garbulsky et al., (2011) and Gamon, (2015) have proposed that the PRI-LUE

82 relationship should vary not only with time scale, but also with vegetation type. According to

83 this view, particularly in annual or deciduous species where green canopy display drives the

84 seasonal photosynthetic response, PRI should be driven primarily by pigment transformations

85 associated with ontogeny and senescence as modulated by environmental conditions, and not the

86 xanthophyll cycle per se. On the other hand, over short time scales in evergreens, where green

87 canopy display changes little, and the chlorophyll (Chl) content is relatively stable, PRI is likely

88 to more closely follow changes in photosynthetic activity mediated in part by rapid operation of

89 xanthophyll cycle. However, across seasons, the PRI changes are more likely to reflect Car/Chl

90 ratio shifts (Wong and Gamon, 2015A and 2015B). This predicted complementary behavior of

91 functionally different vegetation types recently called the "complementarity hypothesis"

92 (Gamon, 2015) offers a framework for evaluating PRI responses across different vegetation 
93 types, but has not yet been fully tested. Since it suggests that the role of pigment pool sizes in

94 driving PRI should vary with vegetation type, condition, and time scale in ecologically

95 significant ways, it provides an ecological and evolutionary framework for testing our

96 understanding of PRI responses across multiple ecosystems and biomes.

97 One way to examine these different perspectives is through the concept of pigment

98 stoichiometry. In many cases, the ratios of Car/Chl pigment pools seem to be largely invariant

99 suggesting accessory light harvesting and its regulation as a predominant functional role for the

100 Car closely associated with the photosynthetic apparatus (Feret et al., 2011). On the other hand,

101 stresses that limit photosynthesis often increase Car/Chl ratios (see Solovchenko, 2010 and

102 references therein). Thus temporal changes in the stoichiometry of plant pigments can give an

103 insight into their functional roles, and stoichiometric variation can be detectable with PRI (Wong

104 and Gamon, 2015A and B). In the context of this study, "stress" refers to any condition that leads

105 to an excess of radiation beyond what can be used for photosynthesis. Conditions leading to

106 such stress can include temperature extremes, drought, or nutrient stress. A well-documented

107 short-term response of leaves to stress is the conversion of violaxanthin to zeaxanthin affecting

108 PRI. In the long term, leaves respond to stress by changing the pigment levels and ratios that we

109 refer to as constitutive changes.

110 A further reason for examining the PRI-pigment relationship is that the LUE model (a

111 primary modeling framework for relating PRI to photosynthetic activity) can be parameterized or

112 defined in multiple ways, and this has implications for the interpretation of PRI. For example,

113 some authors have defined the LUE based on incident radiation, and some on absorbed radiation,

114 while others define it solely on the radiation absorbed by green (photosynthetic) canopy material

115 (see references in Gitelson and Gamon, 2015). These different definitions can have significant 
116 consequences for the conclusions that can be drawn regarding the function of PRI, a topic that

117 has added to the confusion in the PRI literature. Changes in canopy structure and/or

118 pigmentation occurring over a growing season, can lead to the conclusion that LUE, defined or

119 measured via PRI, is changing seasonally, when actually these changes in apparent LUE are

120 being driven by gradual changes in canopy structure and pigmentation associated with plant

121 development, stress acclimation or senescence, among other factors. Functionally, these changes

122 in PRI due to pigment pools and canopy structure are distinct from activity of the xanthophyll

123 cycle that is associated with PSII LUE or non-photochemical quenching (NPQ), and they may or

124 may not coincide with this activity. Without further information to constrain the problem, PRI by

125 itself cannot resolve these underlying mechanisms.

126 The goal of this study was to quantify the relationships between leaf level PRI and pigment

127 pools, focusing on the constitutive role of pigments in influencing PRI over seasonal or

128 ontogenetic time frames. A growing body of evidence suggests a primary role for pigment pools

129 in driving the seasonal PRI responses. However, most studies have been done in evergreens and

130 very few (if any) papers have explored the link between pigment (chlorophyll, carotenoid and

131 anthocyanin) contents and their composition in deciduous species and managed crops (see

132 Garbulsly et al., 2011 for review). The purpose was to re-evaluate the role of PRI as an indicator

133 of pigment (chlorophyll, carotenoid and anthocyanin) pools, and hence photosynthetic activity, 134 across a range of tree and crop species.

136 2. Methods

137 Plant material. Juvenile, mature and senescent leaves of Norway maple (Acer platanoides L.)

138 and horse chestnut (Aesculus hippocastanum L.) were collected in a park at Moscow State 
139 University campus in the spring, summer and fall of 1992-2000 (Table 1). Second-flush beech

140 (Fagus sylvatica L.) leaves grown on the University of Karlsruhe campus were taken in August

141 and September 1996. Leaves were visually selected according to their difference in color. Leaves

142 healthy and homogeneous in color without anthocyanin pigmentation or visible symptoms of

143 damage were used in the experiments (Table 1, details are in Gitelson et al., 2003).

144 Anthocyanin-containing leaves of Norway maple were collected in the park at Moscow State 145 University campus (1992-2000) in spring and fall. In maple, the red pigmentation determined by

146 the presence of anthocyanins was especially expressed during cold seasons. The anthocyanin

147 pigmentation was normally observed in sunlit leaves whereas shaded leaves lacked the

148 anthocyanin pigmentation being green to yellow in color (details are in Gitelson et al., 2001).

149 Growing conditions of tree species were typical for these species in the regions - Moscow,

150 Russia (maple and chestnut), and Karlsruhe Germany (beech).

151 Maize (Zea mays L.) and soybean (Glycine max L.) leaves (Table 2) were collected in

152 irrigated and rainfed sites at Mead Nebraska (USA) in 2003 through 2005 with varying weather

153 conditions. Leaves were collected across the growing season in maize and in the middle of the

154 reproductive stage in soybean when wide variability of pigment content occurred. To study

155 temporal change in leaf pigment content, leaves were collected across the growing season at the

156 rainfed maize site in 2003 with a period of drought lasted from the $190^{\text {th }}$ through $220^{\text {th }}$ day of

157 year, DOY (Peng et al., 2011). For more detail see Gitelson et al., 2005; 2006).

158 Pigment analysis. The leaf pigment content was determined from the same leaf samples that

159 were used for reflectance measurement. Leaf disks were cut from the leaves and extracted with

$160100 \%$ acetone or methanol by grinding the disks in a mortar and a pestle. The pigment

161 homogenates were centrifuged for 3-5 min at $3000 \mathrm{~g}$ in glass tubes to obtain the fully transparent 
162 supernatant which was immediately assayed spectrophotometrically. Specific absorption

163 coefficients of Chl $a$, Chl $b$ and total carotenoids reported by Lichtenthaler (1987) were used.

164 The accepted average molecular weight of carotenoids was $540 \mathrm{~g} \mathrm{~mol}^{-1}$.

165 Anthocyanin content was determined after extract acidification with concentrated $\mathrm{HCl}$.

166 Absorbance at $530 \mathrm{~nm}$ was corrected for pheophytin contribution: pheophytins $a$ and $b$ were

167 obtained from corresponding chlorophylls (Fluka Chemie AG) and their absorption coefficients

168 at $530 \mathrm{~nm}$ in acid methanol were found to be 8.17 and $6.35 \mathrm{mM}^{-1} \mathrm{~cm}^{-1}$, respectively.

169 Anthocyanin absorption coefficient of $30 \mathrm{mM}^{-1} \mathrm{~cm}^{-1}$ at $530 \mathrm{~nm}$ (Strak Wray 1989) was used

170 (details in Gitelson et al., 2001). Pigment content was expressed on leaf area basis in $\mathrm{mg} \mathrm{m}^{-2}$

171 (Tables 1 and 2).

172 Reflectance measurements. Adaxial reflectance $(\rho)$ spectra of the leaves were taken in a spectral

173 range between 400 and $800 \mathrm{~nm}$ with a spectral resolution of $2 \mathrm{~nm}$ with a Hitachi 150-20

174 spectrophotometer equipped with a $150 \mathrm{~mm}$ integrating sphere for reflectance measurements

175 (maple and chestnut leaves), a Shimadzu 2101 PC spectrophotometer equipped for reflectance

176 measurements with an integrating sphere (beech leaves). Maize and soybean leaves' reflectance

177 spectra were measured using an Ocean Optics radiometer (US92000, Ocean Optics, Dunedin FL,

178 USA) with an Ocean Optics LS-1 light source with a Mini Leaf Clip (UNI501, PP Systems,

179 Amesbury MA, USA) with a 2.3-mm diameter bifurcated fiber-optic attached to both radiometer

180 and light source. In each leaf at least five reflectance spectra were measured and median value of

181 reflectance was used for analyses. A 99\% reflectance standard (Spectralon, LabSphere, North

182 Sutton NH, USA) was scanned before and after each leaf measurement. The reflectance was

183 calculated as the ratio of upwelling leaf radiance to the upwelling radiance of the standard. 
186 Table 1. Pigment content (in $\mathrm{mg} \mathrm{m}^{-2}$ ) and $\mathrm{Car} / \mathrm{Chl}$ ratio in tree leaves studied; $n$ is the number of 187 leaves in each data set.

\begin{tabular}{|c|c|c|c|}
\hline & $\min$ & $\max$ & mean \\
\hline \multicolumn{4}{|c|}{ Chestnut $n=27$} \\
\hline Chl & 117.90 & 562.50 & 346.39 \\
\hline Car & 30.85 & 83.20 & 55.30 \\
\hline $\mathrm{Car} / \mathrm{Chl}$ & 0.09 & 0.46 & 0.20 \\
\hline
\end{tabular}

Beech $n=37$

$\begin{array}{lccc}\text { Chl } & 144.45 & 675.00 & 431.62 \\ \text { Car } & 43.51 & 137.18 & 91.55 \\ \text { Car/Chl } & 0.15 & 0.44 & 0.24\end{array}$

Maple (AnC-free) $n=61$

188

\begin{tabular}{lccc} 
Chl & 3.00 & 570.00 & 189.16 \\
Car & 14.60 & 82.60 & 41.18 \\
Car/Chl & 0.09 & 0.50 & 0.16 \\
\multicolumn{4}{c}{ Maple (AnC-containing) $n=23$} \\
Chl & 103.2 & 399.8 & 268.7 \\
Car & 29.81 & 101.9 & 55.67 \\
Anth & 10.73 & 298.34 & 130.1 \\
Car/Chl & 0.11 & 0.69 & 0.21 \\
\hline
\end{tabular}

189 Table 2. Pigment content (in $\mathrm{mg} \mathrm{m}^{-2}$ ) and ratio in the crop leaves studied; $n$ is the number of 190 leaves in data set.

191

\begin{tabular}{|c|c|c|c|}
\hline & $\min$ & $\max$ & mean \\
\hline \multicolumn{4}{|c|}{ Maize $n=20$} \\
\hline $\mathrm{Chl}$ & 2.80 & 775.21 & 229.21 \\
\hline Car & 45.53 & 143.16 & 64.61 \\
\hline Car/Chl & 0.18 & 18.84 & 0.24 \\
\hline \multicolumn{4}{|c|}{ Soybean $n=20$} \\
\hline $\mathrm{Chl}$ & 105.33 & 859.05 & 424.01 \\
\hline Car & 43.81 & 174.46 & 88.30 \\
\hline $\mathrm{Car} / \mathrm{Chl}$ & 0.19 & 0.45 & 0.21 \\
\hline
\end{tabular}

192

193 


\section{Results and Discussion}

3.1 Chlorophyll vs. carotenoid content relationship

Three main pigment groups, Chl, Car, and $\mathrm{AnC}$, affect reflectance at the two wavelengths used for PRI. To quantify the effect of the pigments on PRI, we firstly analyzed PRI-Chl and PRI-Car relationships in AnC-free leaves. It is often difficult to discern the specific behavior of these relationships against the background of an inherently close correlation between $\mathrm{Chl}$ and Car content, because Chl and primary (photosynthetic) Car are bound in the pigment-protein complexes of the photosynthetic apparatus according to a conserved stoichiometry that is often species-specific At the same time, absolute amounts of these pigments and their ratios can be adjusted within genetically programmed limits in response to environmental stimuli. By contrast, pigments that do not participate in assimilation of light energy (exemplified by secondary Car) are not so strictly controlled genetically and may vary over a much wider range e.g. during senescence and/or under stress (Green and Durnford, 1996; Young, 1993).

For the species studied, the Chl-Car relationships were very close $(\mathrm{p}<0.001)$, with $\mathrm{R}^{2}$ above 0.7 for leaves of the three tree species and above 0.94 for both crops (Fig. 1 for beech and maize). A similar close relationship $\left(\mathrm{R}^{2}=0.86\right)$ was found in seventeen independent datasets including a wide range of leaf spectral, chemical, and structural properties collected all over the world (Feret et al., 2011). This largely conserved stoichiometry has led to the perspective of an invariant Chl/Car ratio in many modeling studies (Jacquemoud and Baret, 1992).

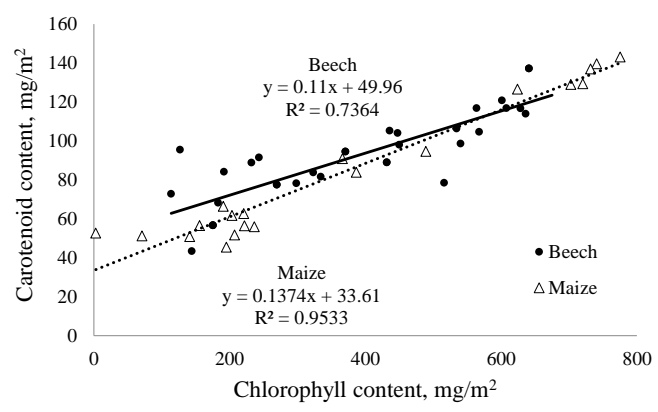


214 Figure 1. Relationships between carotenoid and chlorophyll contents in beech and maize leaves.

PRI uses reflectance at $531 \mathrm{~nm}$ in the so-called green edge and at $570 \mathrm{~nm}$ in the green

217 range of the spectrum. The green edge is a transition region where in situ absorption by Chl $a$

218 and $b$ and different Car drops sharply from a high value at $480 \mathrm{~nm}$ to an almost negligible

219 amount above $531 \mathrm{~nm}$ (Gitelson et al., 2002; Feret et al., 2016). Reflectance in the green edge

220 range is governed by $\mathrm{Chl} a, \mathrm{Chl} b, \mathrm{Car}$ and $\mathrm{AnC}$ absorption. In some studies, the reflectance at

$221570 \mathrm{~nm}$ was invariant with respect to Car content due to very small (if any) Car absorption

222 (Lichtenthaler, 1987; Gitelson et al., 2002; Feret et al., 2008; 2011, 2016). Thus, it was expected

223 that PRI in AnC-free leaves would be affected by both Car and Chl pigments, and we found

224 significant PRI-Chl and PRI-Car relationships in all tree and crop leaves studied (Fig. 2 for beech

225 and maize). PRI-Chl relationships were closer than PRI-Car ( $\mathrm{R}^{2}$ was 0.73 for Chl vs. 0.47 for Car

226 in beech, and 0.62 vs. 0.53 in maize, respectively; $\mathrm{p}<0.001)$. Multiple regression analysis of

227 PRI vs. Car and Chl relationships showed that both pigments together were responsible for more

228 than $74 \%$ of PRI variation in maize and $76 \%$ in beech.

229

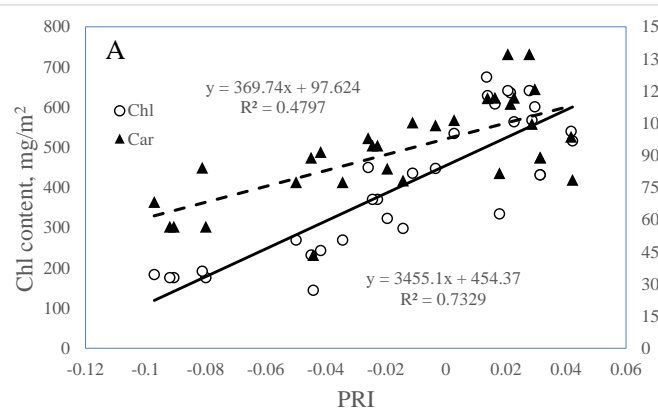

PRI

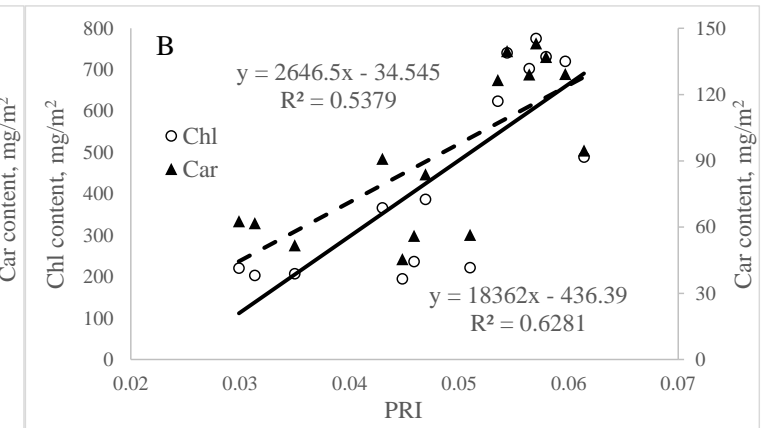

231 Figure 2. Relationships between PRI and pigment contents in beech (A) and maize (B) leaves. 
To illustrate how the deviation of points from Chl-PRI and Car-PRI relationships (Fig. 2) is 234 affected by the close Chl-Car relationships (Fig. 1), we plotted residuals of the PRI-Chl relationship versus residuals of the PRI-Car relationship (Fig. 3). The relationships between residuals were very close for both tree and crop leaves and, as for the Chl-Car relationship (Fig. 1), $R^{2}$ was higher for crop than for tree leaves. Relationships for tree and crop leaves were very 238 close to each other with $\mathrm{R}^{2}$ above 0.79 for tree leaves and above 0.95 for crops.

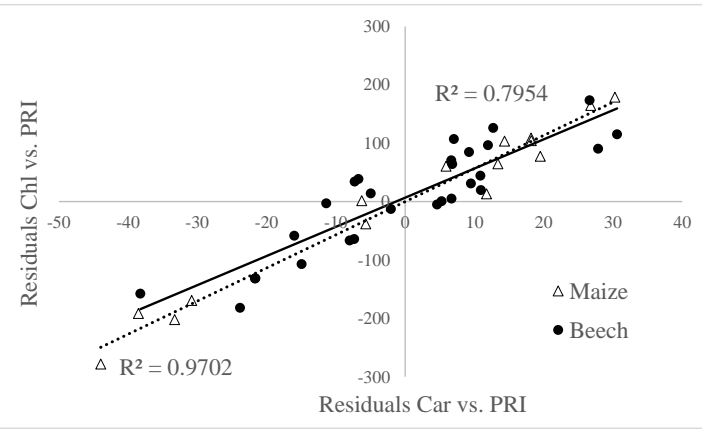

240 Figure 3. Relationships between residuals of Chl vs. PRI and Car vs. PRI relationships for beech 241 and maize leaves.

The reasoning for such close relationships may be the tightly related $\mathrm{Chl}$ and Car contents

244 that often vary synchronously during ontogeny and senescence. Since both pigments affect 245 reflectance at $530 \mathrm{~nm}$, PRI-pigment relationships are quite scattered (Fig. 2), but the deviations 246 from best-fit functions (i.e., the residuals) of Chl-PRI and Car-PRI relationships are inherently 247 linked. In other words, if a certain Chl-PRI data point departs from a linear trend, the 248 corresponding Car-PRI data point has a high probability of diverging from its corresponding 249 linear trend by a certain value which is 'hardcoded' by the close Car-Chl relationship. The 250 absolute deviation of these departures may be different, but their ratios show a similar strength as 251 the Chl-Car relationship. Thus, the close relationship shown in Fig. 3 is an inevitable 252 consequence of close Car-Chl relationship for these particular species and growing conditions 
253 (Fig. 1). However, a close Car-Chl relationships might not necessarily hold for the wide range of

254 leaf traits, species, or conditions existing in nature or under all cultivation conditions. As could

255 be seen in Fig 1, the tree leaves studied did not show as tight a stoichiometry as crop leaves,

256 suggesting that ontogenetic and environmental factors may be at play (Wright et al., 2004),

257 suggesting that pigment ratios were responding in a different way to the tight stoichiometric

258 ratios seen in crops, as further discussed below.

259

260

3.2 Departure from a strong stoichiometric relationship

261

Reasons for deviations from the main Car/Chl trend could be divided, based on time scale,

262 into life-long and short-term responses, and many of these reflect a leaf's response to the amount

263 of excess energy (Björkman and Demmig-Adams 1994, Gamon 2015). Over longer periods,

264 acclimation to elevated solar irradiances have been known to increase the Car/Chl ratio due to

265 decline in Chl content against the background of Car retention (Young, 1993). Shifts in the

266 Car/Chl ratio over long time scales in response to environmental conditions (Wong and Gamon,

267 2015A), or during senescence-induced Car transformation (Merzlyak et al., 1999; Gamon et al.,

268 2001), lead to gradual spectral changes that influence the PRI signal. Furthermore, differences in

269 the amount of excess radiation can influence the composition of distinct Car having slightly

270 different spectral properties (Britton, 1995) and thus affect the PRI-Car relationship. Thus, the

271 change in the proportion between light-harvesting (e.g. lutein) and photoprotective Car (e.g.

272 violaxanthin cycle xanthophylls) might also displace both the Car/Chl ratio and PRI.

273 Over long time spans, additional disturbing factors include the accumulation of so called

274 "secondary carotenoids" and/or acclimatory rearrangements of the pigment apparatus. In contrast

275 to "primary" or photosynthetic Car, which are tightly linked with photosynthetic apparatus 
276 (PSA), being involved in light harvesting and photoprotection, secondary Car are structurally and 277 functionally uncoupled from PSA (Young, 1993, Solovchenko, 2010). As a result, secondary Car 278 do not obey the strict stoichiometry linking the primary Car and Chl contents, and this appears to 279 be the case for the deciduous tree leaves. In contrast, very close Car-Chl relationships evident in 280 crops (Fig. 1) indicate leaves with a negligible content of secondary Car. This situation is clearly 281 illustrated by the temporal behavior of Chl content, Car/Chl ratio and PRI as a function of day of 282 year, DOY (Fig. 4) in rainfed maize site during 2003 with period of drought around DOY 180283200 (Peng et al., 2013). The PRI closely followed the increase in Chl early in the growing 284 season as well as the decrease of $\mathrm{Chl}$ in the reproductive stage beyond DOY 210. However, in a 285 period of decrease in Chl content due to water deficiency (DOY 180-205) PRI did not follow Chl 286 content. Due to the very close relationship between $\mathrm{Chl}$ and Car $\left(\mathrm{R}^{2}\right.$ above $\left.0.97, \mathrm{p}<0.0001\right)$, the 287 Car/Chl ratio in this period of stress increased very slightly. It underlines the limitation of using 288 seasonal PRI changes as an indicator of plant stress in cases where there is a strict stoichiometry 289 of Chl and Car.

290 291 292 293 294 295 296
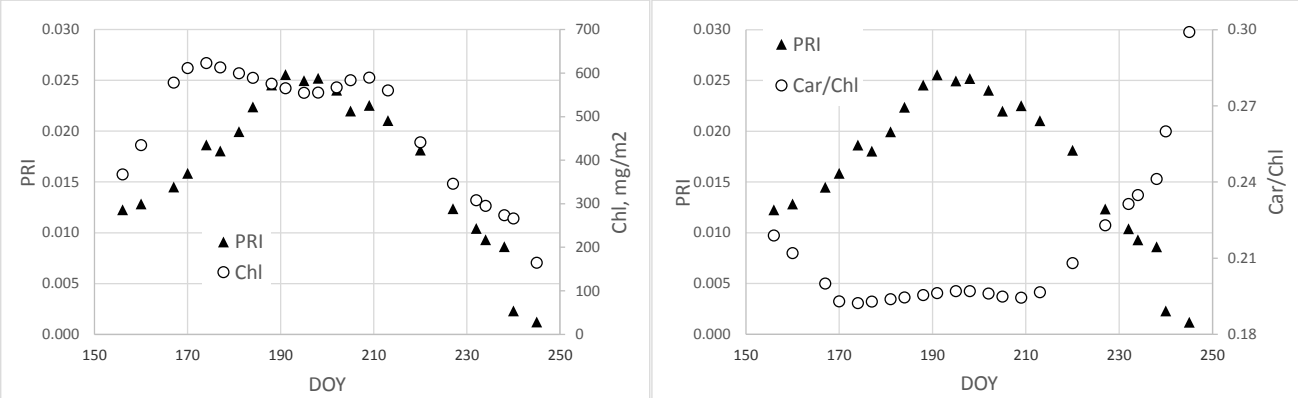

Figure 4. Temporal change of PRI and Chl content (A) and PRI and Car/Chl ratio (B) in leaves collected at maize rainfed site in year 2003 with drought event between day of year (DOY) 190220.

In contrast to crops, where the Car-Chl ratio is conserved, we observed a much weaker CarChl relationship in tree leaves (Fig. 1), most likely due to an increased accumulation of 
298 Steinmüller, 1985) as well as number of other plant species (Merzlyak et al., 2005, Han et al., 299 2003, Hormaetxe et al., 2005) often contain lipid inclusions serving as a depot for secondary Car.

300

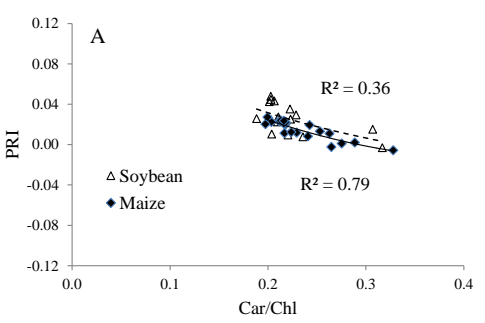

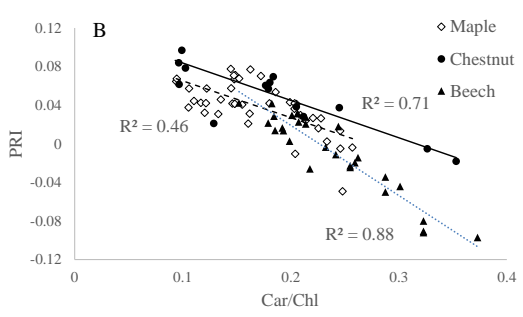

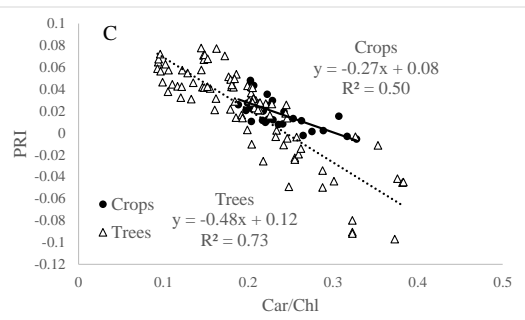

301

Figure 5. Relationships between PRI and Car to Chl ratio in crop (A) and tree (B) leaves. Also shown are summary relationships comparing all crops and trees investigated (C) where PRI 303 explained $73 \%$ of $\mathrm{Car} / \mathrm{Chl}$ variation in crops and $50 \%$ in trees $(\mathrm{p}<0.05)$.

Due to the close relationship between the residuals (Fig. 3), it is not surprising that PRI was found to be closely related to the Car/Chl ratio (Fig. 5). As a result of the strong stoichiometric 307 relationship between Chl and Car for crops (Fig. 1), the range of PRI variation was narrow and 308 the slope of the PRI vs. Car/Chl relationship was very small (Fig. 5A). By contrast, the PRI vs. 309 Car/Chl relationship for tree leaves illustrates how the departure from this strong stoichiometric relationship affects the sensitivity of PRI to Car/Chl ratio (Fig. 5B). The PRI sensitivity to

$311 \mathrm{Car} / \mathrm{Chl}$ in tree leaves was higher than in crops, which emerges as a steeper slope of the PRI vs.

312 Car/Chl relationship.

In two crops (soybean and maize) having different photosynthetic pathways $\left(\mathrm{C}_{3} \mathrm{vs} . \mathrm{C}_{4}\right)$ and

314 related leaf structure, the slopes of PRI vs. Car/Chl relationships were quite close to each other.

315 In contrast, the slopes of PRI vs. Car/Chl relationships for three unrelated tree species were 316 different depending on the closeness of Car vs. Chl relationships and the dynamic range of each 317 pigment content (Fig. 5B). Despite these differences, a significant uniform the PRI vs. Car/Chl 
318 relationship was formed for three tree species with PRI explaining more than $73 \%(\mathrm{p}<0.05)$ of

319 Car/Chl variation (Fig. 5C). The same was the case for crop leaves also forming a uniform (but

320 distinct from the trees leaves with two-fold smaller slope) significant PRI vs. Car/Chl

321 relationship where PRI explained about 50\% (p < 0.05) of Car/Chl variation (Fig. 5C).

322 Rahimzadeh-Bajgiran et al. (2012) evaluated PRI for assessing plant photosynthetic

323 performance throughout the plant life cycle in eggplant. PRI was significantly correlated with

324 plant pigments estimated via vegetation indices (VI) retrieved from leaf reflectance. They found

325 that photosynthetic capacity and steady-state PRI could be better explained by Chl content than

326 by xanthophylls, arguing that PRI reflects pigment content over long time spans. To test how the

327 VIs used in their study relate to leaf pigment content, we calculated the VIs using measured

328 reflectance and compared them to analytically determined pigment contents. The relationships

329 were very close with $\mathrm{p}<0.0001$ (Fig. S1). Thus, our findings on the dominant role of pigment

330 pools over long time spans closely correspond to Rahimzadeh-Bajgiran et al., (2012) results

331 taken at different species.

Thus, in AnC-free tree and crop leaves PRI was related to both Chl and Car contents, but

333 much more closely to the Car/Chl ratio. The sensitivity of PRI to Car/Chl depends strongly on

334 the fidelity (i.e. stoichiometry) of the Chl/Car relationship. These processes might not all be

335 immediately or equally linked with LUE and this may confound the interpretation of PRI in

336 remote sensing studies that have often assumed a single mechanism of PRI response linked to the

337 xanthophyll cycle. These findings illustrate the importance of assessing leaf-level pigment levels

338 when evaluating PRI or other similar vegetation indices. Variation in PRI-LUE responses in

339 remote sensing studies spanning dates or species could in fact reflect different functional roles of

340 the pigments influencing PRI, leading to misinterpretation of the underlying function. Profound 
341 changes in Car/Chl ratio and Car composition take place during ontogenesis during the

342 maturation and senescence of the leaf photosynthetic apparatus (Merzlyak et al., 1998,

343 Lichtenthaler and Babani, 2004). This trend of Car/Chl ratio is genetically programmed and

344 further modulated by acclimation to long-term stresses (Solovchenko et al., 2006, Lichtenthaler

345 and Babani, 2004). Similarly, Car/Chl ratios, and hence PRI values, vary with canopy position

346 and irradiance (e.g., Hilker et al., 2008; Gamon and Berry, 2012). These processes reflect

347 ontogenetic and acclimatory responses to prevailing conditions, and obviously exert a dominant

348 contribution to the changes in PRI observed over seasonal timescales. What is less clear (and

349 requires further study) is how closely PRI reflects underlying photosynthetic activity or LUE

350 across this range of pigment responses, and to what degree the varying PRI-LUE relationships

351 often reported in the literature (Nichol et al., 2002, Goerner et al., 2011) reflect these different

352 mechanisms.

Behavior of PRI in AnC-containing leaves was very different from that in AnC-free leaves.

354 Anthocyanins absorb in situ in a wide range around $550 \mathrm{~nm}$ (Gitelson et al., 2001, Peters and

355 Noble, 2014; Feret et al., 2016) affecting reflectance at both wavelengths used in PRI. In AnC-

356 containing leaves, reflectances at 530 and $570 \mathrm{~nm}$ are governed mainly by AnC absorption (i.e.,

357 AnC content). When AnC and Chl contents did not relate closely (as in our data), there was no

358 significant relationship between PRI and any pigment (Chl, Car and AnC) content as well as

359 with Car/Chl (Fig. S2). The disturbance of the close Car/Chl relationship upon appearance of

360 AnC can be explained by a strong photoprotective effect of anthocyanins (Hatier and Gould,

361 2009, Gould, 2004). When AnC accumulated in appreciable amounts, it takes over the

362 photoprotective function of Car resulting in a weakening of its tight relationships with PRI. The

363 presence of anthocyanins led to a strong interference of AnC absorption with the PRI-Car/Chl 
relationship, and indicates an additional leaf photoprotective strategy from the xanthophyll cycle that is available to some species (e.g. Quercus spp. Gamon and Surfus 1999). Additional studies are needed to further elucidate alternative leaf strategies for photoprotection over different time scales and their overall effects on leaf optical signals.

\section{Conclusions}

Analysis of seasonal constitutive changes in foliar pigment content and composition of three tree species and two crop species lead to the following conclusions.

- $\quad$ In anthocyanin-free leaves, PRI related to both total Chl and Car content. Each pigment (Chl and Car) content explained from 43 to $73 \%$ of PRI variation. Both pigments together were responsible for more than $74 \%$ of PRI variation.

- In the tree and crop leaves, PRI was closely related to the $\mathrm{Car} / \mathrm{Chl}$ ratio over long time spans, most likely affecting physiological and ontogenetic factors.

- $\quad$ PRI vs. Car/Chl relationships for leaves of three different tree species formed a significant uniform relationship with PRI explaining more than $73 \%$ of $\mathrm{Car} / \mathrm{Chl}$ variation.

- The crop leaves displayed a strict stoichiometric relationship between $\mathrm{Chl}$ and $\mathrm{Car}$, and also formed a significant PRI vs. Car/Chl relationship (distinct from that observed in the trees) where PRI explained about 50\% of Car/Chl variation.

- The sensitivity of PRI to $\mathrm{Car} / \mathrm{Chl}$ in the tree leaves was at least 2-fold higher than in the crops. It was boosted by less close $\mathrm{Chl}$ vs. Car relationships in the tree leaves stemming likely from the increased contribution of secondary carotenoids.

- In anthocyanin-containing leaves, PRI did not clearly relate to any pigment content due to the effect of AnC absorption on reflectance at both PRI wavelengths. This indicates both a confounding effect of anthocyanins on reflectance and an alternative leaf photoprotective strategy.

These findings are consistent with other recent research in other vegetation types indicating that constitutive pigment effects, not the xanthophyll cycle, are the primary cause of PRI variation over long time spans, and illustrate the importance of assessing leaf pigments when evaluating vegetation indices. Finally, the strong link between leaf-level PRI and Car/Chl, as 
well as PRI and separate Chl and Car contents suggest the need for a more careful evaluation of the relationship between PRI and LUE and photosynthetic activity. In particular, studies that clarify PRI, pigments, and photosynthetic responses over short-term (e.g. diurnal) vs. long-term (e.g. seasonal) time periods for contrasting vegetation types are needed.

\section{Acknowledgements}

This research was supported by Lady Davis and Marie Curie International Incoming Fellowship to AG, and partially supported by the NASA NACP program and the U.S. Department of Energy. We sincerely appreciate the support and use of facilities and equipment provided by the Center for Advanced Land Management Information Technologies (CALMIT) and data from the Carbon Sequestration Program, both at the University of Nebraska-Lincoln. AS acknowledges the financial support of Russian Scientific Foundation (grant \# 14-50-00029). JG acknowledges support from Alberta Innovates Technology Futures (AITF) and the Natural Sciences and Engineering Research Council (NSERC), Canada.

\section{References}

Britton, G. (1995). UV/visible spectroscopy. In G. Britton, S. Liaaen-Jensen, \& H. Pfander (Eds.), Carotenoids (pp. 13-62). Basel: Birkhauser Verlag.

Bilger, W., Björkman, O., \& Thayer, S. S. (1989) Light-induced spectral absorbance changes in relation to photosynthesis and the epoxidation state of xanthophyll cycle components in cotton leaves. Plant Physiology, 91, 542-551.

Björkman, O., and Demmig-Adams, B. (1994) Regulation of photosynthetic light energy capture, conversion, and dissipation in leaves of higher plants, in: Ecophysiology of Photosynthesis, edited by: Schulze, E.-D., Caldwell, M. M., and Pospisilova, J., Springer, Berlin Heidelberg New York, pp. 27-47. 
Ciganda, V., Gitelson, A.A. and Schepers, J. (2009). Non-destructive determination of maize leaf and canopy chlorophyll content. Journal of Plant Physiology, 166,157-167.

Féret, J-B., Gitelson, A.A., Noble, S.D. and Jacquemoud, S. (2016). PROSPECT-D: modeling leaf optical properties through a complete lifecycle. Submitted to Remote Sensing of Environment.

Féret, J-B., François, C., Gitelson, A.A., Barry, K.M., Panigada, C., Richardson, A.D., and Jacquemoud, S., (2011). Optimizing spectral indices and chemometric analysis of leaf chemical properties using radiative transfer modeling, Remote Sensing of Environment, $115,2742-2750$.

Feret, J.-B., François, C., Asner, G. P., Gitelson A.A., Martin, R.E., Bidel, L.P.R., Ustin, S.L., le Maire, G., and S. Jacquemoud. (2008). PROSPECT-4 and 5: Advances in the Leaf Optical Properties Model Separating Photosynthetic Pigments, Remote Sensing of Environment, 112, 3030-3043, doi:10.1016/j.rse.2008.02.012

Filella, I., Porcar-Castell, A., Munne-Bosch, S., Back, J., Garbulsky, M. F., and Peñuelas, J. (2009). PRI assessment of long-term changes in carotenoids/chlorophyll ratio and shortterm changes in de-epoxidation state of the xanthophyll cycle, International Journal of Remote Sensing, 30, 4443-4455, doi: 10.1080/01431160802575661,.

Hilker, T., Coops, N.C., Hall, F.G., Black, T.A., Wulder, M.A., Nesic, Z., \& Krishnan, P. (2008). Separating physiologically and directionally induced changes in PRI using BRDF models. Remote Sensing of Environment, 112, 2777-2788.

Gamon, J.A. (2015). Optical sampling of the flux tower footprint. Biogeosciences, 12, 45094523. doi:10.5194/bg-12-4509-2015 
Gamon, J.A., Berry, J.A. (2012). Facultative and constitutive pigment effects on the Photochemical Reflectance Index (PRI) in sun and shade conifer needles. Israel Journal of Plant Sciences, 60, 85-95. DOI: 10.1560/IJPS.60.1-2.85

Gamon, J.A., Filella, I., Peñuelas, J. (1993). The dynamic 531-nanometer $\Delta$ reflectance signal: a survey of twenty angiosperm species. Yamamoto HY, Smith CM (Eds). Photosynthetic Responses to the Environment. American Society of Plant Physiologists, Rockville. pp. 172-177.

Gamon, J.A., Peñuelas, J., Field, C.B. (1992). A Narrow-Waveband Spectral Index that Tracks Diurnal Changes in Photosynthetic Efficiency. Remote Sensing of Environment, 41, 3544.

Gamon, J.A., Field, C.B., Fredeen, A.L., Thayer, S. (2001). Assessing photosynthetic downregulation in sunflower stands with an optically-based model. Photosynthesis Research, 67,113-125.

Gamon, J.A., Serrano, L., and Surfus, J. (1997). The photochemical reflectance index: an optical indicator of photosynthetic radiation use efficiency across species, functional types, and nutrient levels. Oecologia 112(4), 492-501.

Gamon, J.A., Surfus, J.S. (1999). Assessing leaf pigment content and activity with a reflectometer. New Phytologist, 143, 105-117.

Garbulsky, M. F., Peñuelas, J., Gamon, J., Inoue, Y., and Filella, I. (2011). The photochemical reflectance index (PRI) and the remote sensing of leaf, canopy and ecosystem radiation use efficiencies: A review and meta-analysis, Remote Sensing of Environment, 115, 281297, doi:10.1016/j.rse.2010.08.023, 2011. 
462 Garrity, S. R., Eitel, J. U. H., and Vierling, L. A. (2011). Disentangling the relationships

463

464

465

466

467

468

469

470

471

472

473

474

475

476

477

478

479

480

481

482 between plant pigments and the photochemical reflectance index reveals a new approach for remote estimation of carotenoid content, Remote Sensing of Environment, 115, 628635, doi:10.1016/j.rse.2010.10.007.

Gitelson A.A., Gamon, J.A. (2015). The need for a common basis for defining light-use efficiency: implications for productivity estimation. Remote Sensing of Environment 156, 196-201.

Gitelson, A.A., and Merzlyak, M. N. (1997). Remote Estimation of Chlorophyll Content in higher Plant Leaves. International Journal of Remote Sensing, 18(12), 291-298.

Gitelson, A.A., Merzlyak, M.N., and Chivkunova, O.B. (2001). Optical properties and nondestructive estimation of anthocyanin content in plant leaves, Photochemistry and Photobiology, 74(1), 38-45.

Gitelson, A.A., Zur, Y., Chivkunova, O.B. and Merzlyak, M.N. (2002). Assessing Carotenoid Content in Plant Leaves with Reflectance Spectroscopy, Photochemistry and Photobiology, 75(3), 272-281.

Gitelson, A.A., Gritz, U. and Merzlyak M.N. (2003). Relationships between leaf chlorophyll content and spectral reflectance and algorithms for non-destructive chlorophyll assessment in higher plant leaves. Journal of Plant Physiology, 160(3), 271-282.

Gitelson, A.A., Viña, A., Rundquist, D.C., Ciganda, V., Arkebauer, T.J. (2005). Remote Estimation of Canopy Chlorophyll Content in Crops, Geophysical Research Letters, 32, L08403, doi:10.1029/2005GL022688 
483 Gitelson, A. A., Keydan, G. P. and Merzlyak, M. N. (2006). Three-band model for noninvasive

484

485

486

487

488

489

490

491

492

493

494

495

496

497

498

499

500

501

502

503

504 estimation of chlorophyll, carotenoids, and anthocyanin contents in higher plant leaves, Geophysical Research Letters, 33, L11402, doi:10.1029/2006GL026457.

Gitelson A.A., Gamon, J.A. and Solovchenko. (2016). Multiple drivers of seasonal change in PRI: Implications for photosynthesis. 2. Canopy level. Submitted to Remote Sensing of Environment.

Goerner A, Reichstein M, Tomelleri E, Hanan N, Rambal S, Papale D, Dragoni D, Schmullius C. (2011). Remote sensing of ecosystem light use efficiency with MODIS-based PRI. Biogeosciences, 8, 189-202.

Gould, K. (2004). Nature's Swiss army knife: the diverse protective roles of anthocyanins in leaves. Journal of Biomedicine and Biotechnology, 5, 314-320.

Green, B. R., and D. G. Durnford. (1996). The chlorophyll-carotenoid proteins of oxygenic photosynthesis. Annual review of plant biology 47.1, 685-714.

Han, Q., Shinohara, K., Kakubari, Y., \& Mukai, Y. (2003). Photoprotective role of rhodoxanthin during cold acclimation in Cryptomeria japonica. Plant, Cell and Environment, 26, 715-723.

Hatier, J.-H. B., \& Gould K. S. (2009). Anthocyanin function in vegetative organs. In Anthocyanins (pp. 1-19). Heidelberg, New York: Springer Science+Business Media.

Hormaetxe, K., Becerril, J., Fleck, I., Pinto, M., \& Garcia-Plazaola, J. (2005) Functional role of red (retro)-carotenoids as passive light filters in the leaves of Buxus sempervirens L.: increased protection of photosynthetic tissues? Journal of Experimental Botany, 56, 2629-2636. 
Horton, P. (2014). Developments in research on non-photochemical fluorescence quenching: emergence of key ideas, theories and experimental approaches. In B. Demmig-Adams, G. Garab, W. Adams III, and Govindjee (Eds.), Non-photochemical quenching and energy dissipation in plants, algae and cyanobacteria (pp. 73-95). Dordrecht: Springer Netherlands.

Jacquemoud, S., and Baret, F. (1990). PROSPECT: A model of leaf optical properties spectra. Remote Sensing of Environment, 34, 75-91.

Jahns, P., Holzwarth, A. R. (2012). The role of the xanthophyll cycle and of lutein in photoprotection of photosystem II. Biochimica et Biophysica Acta-Bioenergetics, 1817, $182-193$.

Nichol CJ, Lloyd J, Shibistova O, Arneth A, Röser C, Knohl A. Matsubara S, Grace J. (2002). Remote sensing of photosynthetic light-use efficiency of a Siberian boreal forest. Tellus $54 B, 677-687$.

Lichtenthaler, H., \& Babani, F. (2004). Light adaptation and senescence of the photosynthetic apparatus. Changes in pigment composition, chlorophyll fluorescence parameters and photosynthetic activity. In G. Papageorgiou, \& G. Govindjee (Eds.), Chlorophyll a fluorescence. A signature of photosynthesis (pp. 713-736). Heidelberg, New York: Springer.

Maxwell, K., \& Johnson, G. (2000). Chlorophyll fluorescence—a practical guide. Journal of Experimental Botany, 51, 659-668.

Merzlyak, M. N., Gitelson, A. A., Chivkunova, O. B., and Rakitin, V. Y. (1999). Nondestructive optical detection of pigment changes during leaf senescence and fruit ripening. Physiologia Plantarum, 106, 135-141. 
528 Merzlyak, M. N., Solovchenko A. E., Smagin A. I., \& Gitelson A. A. (2005). Apple flavonols

529 during fruit adaptation to solar radiation: spectral features and technique for non-

530 destructive assessment. Journal of Plant Physiology, 162, 151-160.

531 Merzlyak, M., Gitelson, A., Pogosyan, S., Lekhimena, L., \& Chivkunova, O. (1998). Light-

532 induced pigment degradation in leaves and ripening fruits studied in situ with reflectance

533 spectroscopy. Physiologia Plantarum, 104, 661-667.

534 Merzlyak, M., Solovchenko, A., \& Pogosyan, S. (2005). Optical properties of rhodoxanthin

535 accumulated in Aloe arborescens Mill. leaves under high-light stress with special

536 reference to its photoprotective function. Photochemical and Photobiological Sciences, 4,

$537 \quad 333-340$.

538 Rahimzadeh-Bajgiran, P., Munehiro, M., and Omasa, K. (2012). Relationships between the

539 photochemical reflectance index (PRI) and chlorophyll fluorescence parameters and plant

540 pigment indices at different leaf growth stages. Photosynthesis Research, 113, 261-271.

541 Peñuelas, J., Filella, I., Gamon, J.A. (1995). Assessment of photosynthetic radiation-use

542 efficiency with spectral reflectance, New Phytologist 131, 291-296.

543 Peters, R.D., and Noble, S.D. (2014). Spectrographic measurement of plant pigments from 300

544 to 800nm. Remote Sensing of Environment, 148, 119-123. doi:10.1016/j.rse.2014.03.020

545 Sims DA, Gamon JA. (2002). Relationships between leaf pigment content and spectral

546 reflectance across a wide range of species, leaf structures and developmental stages.

$547 \quad$ Remote Sensing of Environment 81, 337-354.

548 Solovchenko, A. E. (2013). Physiology and adaptive significance of secondary carotenogenesis

549 in green microalgae. Russian Journal of Plant Physiology, 60, 1-13. 
550 Solovchenko, A. E., Merzlyak, M. N., \& Pogosyan, S. I. (2010). Light-induced decrease of

551

552

553

554

555

556

557

558

559

560

561

562

563

564

565

566

567

568

569

570

571

572

reflectance provides an insight in the photoprotective mechanisms of ripening apple fruit. Plant Science, 178, 281-288.

Solovchenko, A., Avertcheva, O., \& Merzlyak, M. (2006). Elevated sunlight promotes ripeningassociated pigment changes in apple fruit. Postharvest Biology and Technology, 40, 183189.

Solovchenko, A. (2010). Photoprotection in plants: optical screening-based mechanisms. Heidelberg, New York: Springer.

Steinmüller, D., \& Tevini, M. (1985). Composition and function of plastoglobuli. Planta, 163, 201-207.

Strac, D., Wray, V. Anthocyanins. Harborne, J.B., Dey, P.M. editors. Methods in Plant Biochemistry, Vol. 1. New York: Academic Press; 1989. p. 326-352.

Stylinski, C.D., Gamon, J.A., and Oechel, W.C. (2002). Seasonal patterns of reflectance indices, carotenoid pigments and photosynthesis of evergreen chaparral species. Oecologia, 131, 366-374.

Tevini, M., \& Steinmüller, D. (1985). Composition and function of plastoglobuli. Planta, 163, 91-96.

Wong, C.Y.S., Gamon, J.A. (2015A). Three causes of variation in the Photochemical Reflectance Index (PRI) in evergreen conifers. New Phytologist, 206, 187-195, doi: 10.1111/nph.13159

Wong, C.Y.S., Gamon, J.A. (2015B). The Photochemical Reflectance Index (PRI) provides an optical indicator of spring photosynthetic activation in conifers. New Phytologist, 206, 196-208, doi: 10.1111/nph.13251 
573 Young, A. J. (1993). Factors that affect the carotenoid composition of higher plants and algae. In: A. J. Young, \& G. Britton (Eds.) Carotenoids in photosynthesis (pp. 160-205).

575

$576 \quad$ List of figure captions

577 Figure 1. Relationships between carotenoid and chlorophyll contents in beech and maize leaves.

578 Figure 2. Relationships between PRI and pigment contents in beech (A) and maize (B) leaves.

579 Figure 3. Relationships between residuals of Chl vs. PRI and Car vs. PRI relationships for beech

580 and maize leaves.

581 Figure 4. Temporal change of PRI and Chl content (A) and PRI and Car/Chl ratio (B) in leaves

582 collected at maize rainfed site in year 2003 with drought event between day of year (DOY) 190583220.

584 Figure 5. Relationships between PRI and Car to Chl ratio in crop (A) and tree (B) leaves. Also 585 shown are summary relationships comparing all crops and trees investigated (C) where PRI 586 explained $73 \%$ of $\mathrm{Car} / \mathrm{Chl}$ variation in crops and $50 \%$ in trees $(\mathrm{p}<0.05)$. 